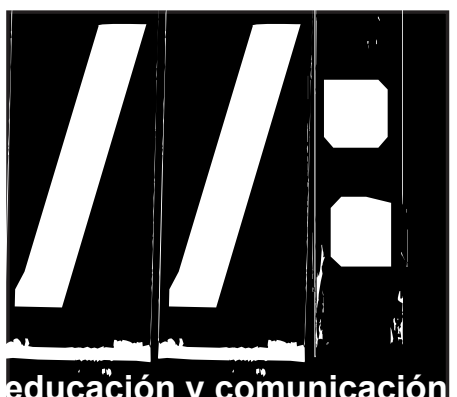

educación y communicación 1: 105-110 Nov. 2010

\section{REDES SOCIALES: CAMBIENTE APTO PARA MENORES?}

[Social Networks: cenvironment for children?]

Autor. Hugo Carrión Gordón

E.mail: hcarrion@imaginar.org

para la Sociedad de la

Información - IMAGINAR

Quito (Ecuador)

Profesor de Postgrado en la Universidad Politécnica

Nacional de Quito

\title{
Resumen:
}

Se analiza la presencia de menores de edad en las llamadas redes sociales. Se presenta una breve caracterización de una de las redes sociales de mayor crecimiento e impacto. El fenómeno de la red facebook es analizado con base a datos estadísticos de sus integrantes. El país de procedencia, la edad y el sexo son algunas de las variables que sirven para analizar las implicaciones de la presencia de niños y adolescentes en las redes sociales. Se mencionan las principales amenazas a las que están expuestos los menores de edad y se concluye con algunas recomendaciones. Palabras claves: Redes sociales, amenazas, intimidación,

\section{Abstract}

We analyze the presence of minors in the social networks. We present a brief characterization of one of the fastest growing social networks and impact. The phenomenon of facebook network is analyzed based on statistical data of its members. The country of origin, age and sex are some of the variables used to analyze the implications of the presence of children and adolescents and social networks. We mention the main threats that children are exposed and we concluded with some recommendations.

Keywords: Social networks, threats, bullying.

(Solicitado: 12-01-10 / Aceptado: 28-09-10) 


\section{II: Pantallas, Educación y Sociedad}

\section{Caracterización}

$\mathrm{E}^{\mathrm{a}}$ fenómeno de internet despegó definitivamente a partir de que la información empezó a ser expuesta en formato hipertextual. El invento inicialmente académico de Tim Berners Lee llamado World Wide Web, empezaría a convertirse en un estándar de información para la presentación de páginas multimedia vinculadas alrededor del mundo. Veinte años más tarde la web ha crecido y nos presenta nuevos desafíos. Desde el año 2004 empezó a acuñarse el término de web 2.0, para referirse a las aplicaciones en las cuales la participación del usuario empieza a ser más preponderante. Plataformas como los blogs, las wikis y las redes sociales empiezan a popularizarse de manera inusitada.

A mediados del año 2010, el mundo ya registra una población conectada a internet cercana al $30 \%$. Es decir cerca de dos mil millones de personas en el mundo tienen acceso a la tecnología de información y comunicación más revolucionaria de los últimos años. Se calcula que al menos la mitad de los usuarios de internet son miembros de redes sociales digitales. Redes como Hi5, myspace, facebook, twitter, linkedin entre otras son las más concurridas y populares. Su evolución ha sufrido cambios significativos y la cantidad de seguidores sin duda depende del lugar geográfico, de los intereses compartidos e incluso de la edad.

En la actualidad la facebook es la red social más estable, con más de quinientos millones de miembros y un crecimiento sostenido que la ubican en el primer lugar del ranking de las redes sociales. Por esta razón el siguiente análisis se centrará en esta red en particular.

Cuando en febrero de 2004 Mark Zuckerberg creó facebook, se centró en un público totalmente universitario, de hecho esta red nace en la Universidad de Harvard y paulatinamente crece a otras universidades. A pesar de la oposición inicial de sus miembros, en septiembre de 2006 facebook se abre a todos los usuarios de internet. A continuación se presenta un breve análisis de algunos países para ilustrar la participación de menores de edad en facebook.

La figura 1, muestra el porcentaje de usuarios de facebook y de internet respecto a la población total de los países representados. En la mayoría de países en desarrollo, la tendencia es que la mayoría de personas que tienen acceso a internet es miembro de esta red social. El caso de Brasil es particular por cuanto facebook tiene poca popularidad, ya que es opacada por Orkut, una red que divide el $90 \%$ de sus miembros entre Brasil, la India y Estados Unidos. 


\section{1/: Redes sociales: ¿Ambiente apto para menores?}

\section{Divulgatio}

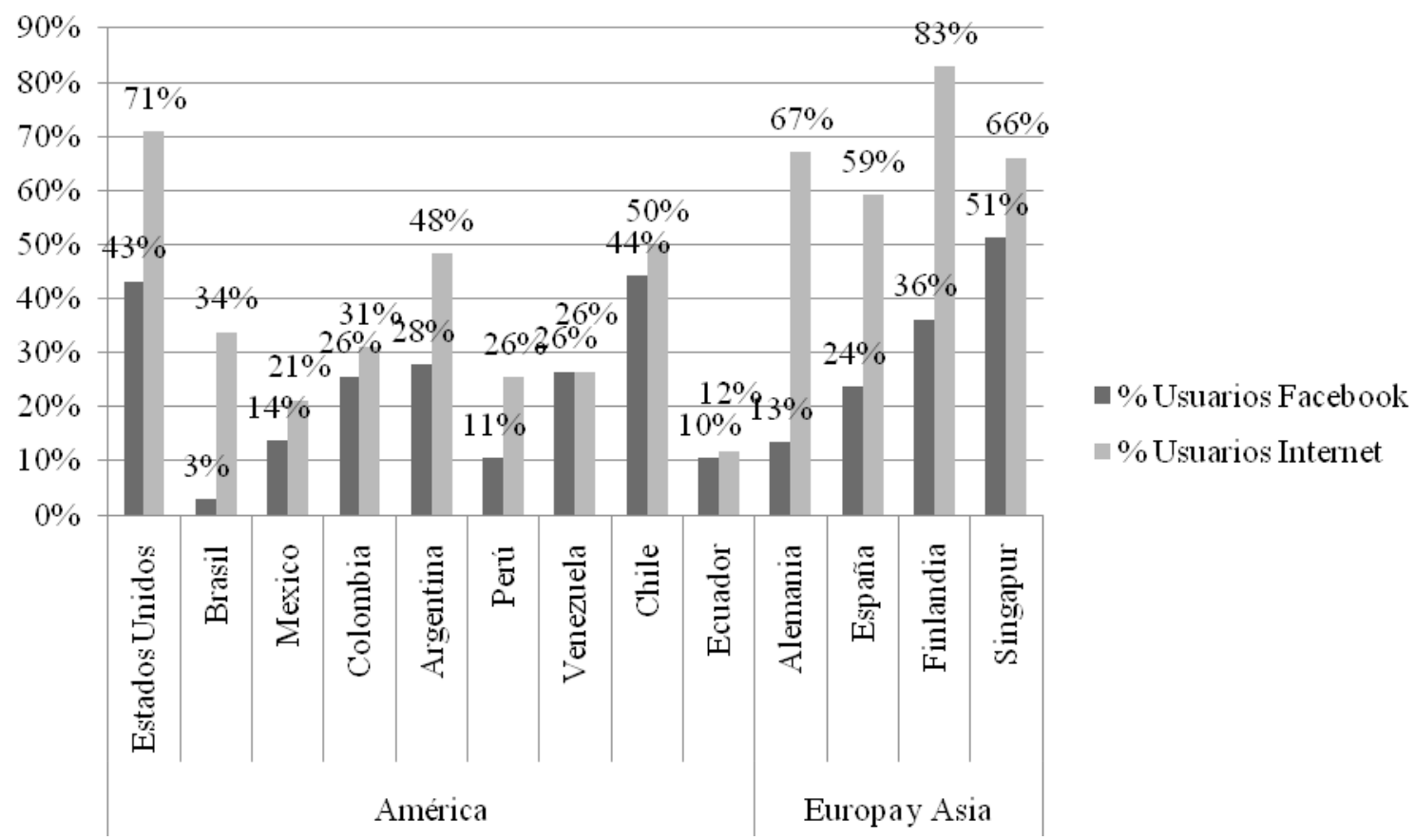

Figura 1: Porcentaje de usuarios de facebook e internet respecto a la población total Fuente Facebook. Datos a septiembre de 2010. Elaboración propia

La participación en redes sociales al parecer no es una actividad que discrimina sexos, la figura 2, muestra el porcentaje de hombres y mujeres que son miembros de facebook. Como se puede apreciar la participación de las mujeres es mayor del $50 \%$ en gran parte de los países representados. 


\section{II: Pantallas, Educación y Sociedad}

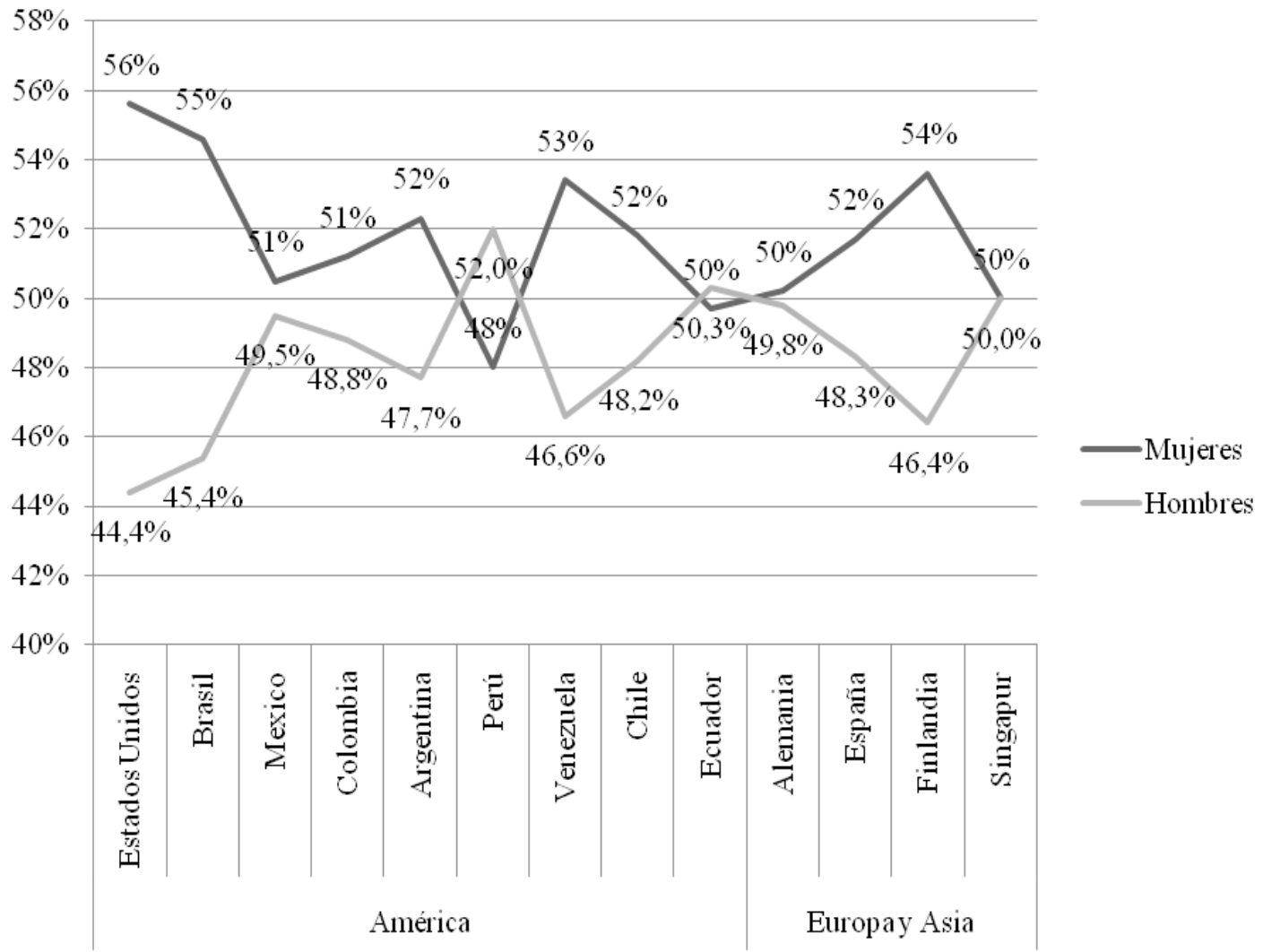

Figura 2: Porcentaje de hombre y mujeres miembros de facebook Fuente Facebook. Datos a septiembre de 2010. Elaboración propia

Para analizar la participación de menores de edad en las redes sociales, se tomaron en cuenta las estadísticas de participación de dos grupos etarios. El primero de menores de 13 años y el segundo de 14 a 17 años. Como se muestra en la figura 3 , la participación de menores de 13 años varía entre un $0,2 \%$ para el caso de España y el 2,2\% para el caso de Ecuador. Mientras que el rango de edad comprendida entre 14 y 17 años, varía entre el $8.7 \%$ en Brasil y el 21,5\% en Colombia. 


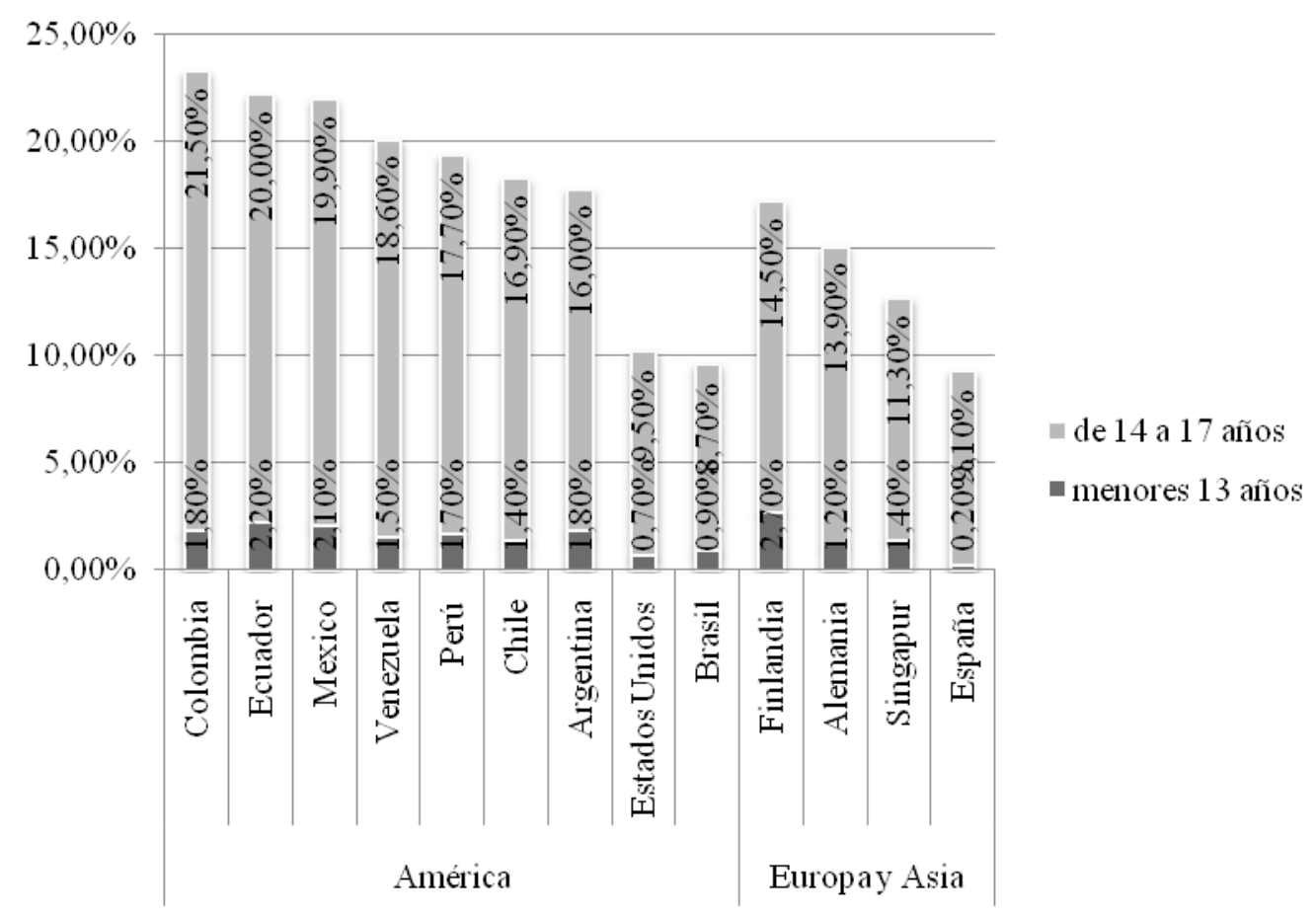

Figura 3: Porcentaje de participación en facebook de menores de edad Fuente Facebook. Datos a septiembre de 2010. Elaboración propia

A pesar de que casi el $40 \%$ de los miembros de facebook corresponden a edades entre 18 y 24 años, no es despreciable el número de menores de edad que participan en ella. Adicionalmente el sexo no parece ser un factor de discriminación, y los países en desarrollo es donde se da una mayor participación de menores de edad.

\section{Amenazas y riesgos para menores en redes sociales}

Sin duda la posibilidad de comunicarnos de manera directa, interactiva e inmediata son algunas de las ventajas de las redes sociales. Sin embargo estas plataformas tecnológicas también son propicias para 
reproducir comportamientos humanos reprochables. La invasión a la privacidad, el "voyerismo" o la intimidación, son potenciados en muchos casos en estos ambientes virtuales. A continuación una breve descripción de los principales riesgos de la participación de menores en redes sociales:

- La información que se publica permanece, si se borra no necesariamente desaparece. Por ello la difusión de información privada tiene repercusiones.

- Tras el uso de seudónimos se podría proteger el anonimato de adultos sin escrúpulos que buscan entablar conversaciones inapropiadas con menores. Estos encuentros pueden conllevar al interés de propiciar contactos físicos o reales.

- Las amenazas, las burlas y el hostigamiento entre pares, que ocurre en la vida real, tiene un campo fértil en las redes sociales. El llamado" ciberbullyng", es un problema creciente en internet.

- No existe control de contenidos, por lo tanto el acceso a mensajes o páginas con contenido o lenguaje sexual o violento puede ser indiscriminado.

Ante estas amenazas y riesgos, la pregunta que queda flotando es ¿son las redes sociales un ambiente propicio para la participación de menores de edad?

\section{Conclusiones}

Se ensaya las siguientes recomendaciones a manera de conclusiones:

- Es fundamental rescatar el papel pro- tagónico de los padres de familia respecto a la formación en el uso responsable y seguro de Internet y la participación en redes sociales.

- Los centros educativos son un espacio privilegiado para la formación tanto de padres de familia como profesores en lo relacionado con la prevención de los riesgos que enfrentan los menores de edad en ambientes digitales.

- Es de vital importancia formar a niños y adolescentes en el uso responsable de internet. Transmitir que toda acción tiene consecuencias y que la virtualidad no significa impunidad.

- Fortalecer la concepción de la privacidad, el valor de la información personal y las consecuencias de difundirla.

- Los padres de familia y centros educativos deben conocer que existen mecanismos de resolución de conflictos, recursos jurídicos que protegen a niños y adolescentes ante eventuales amenazas generadas en ambientes virtuales o por la participación en redes sociales digitales.

\section{Referencias}

Interpol (2009), Legislation of Interpol member states on sexual offences against children, (http://www.interpol.int/Public/Children/SexualAbuse/NationalLawsold/) (Consultada el 6 de septiembre de 2010)

Memorándum sobre la protección de datos personales y la vida privada en las redes sociales en Internet, en particular de niños, niñas y adolescentes. (2009) Memorándum de Montevideo (http://www. iijusticia.org/Memo.htm) (Consultada el 9 de septiembre de 2010) 